\title{
Un nouveau Paracamallanus (Nematoda; Camallanidae), chez un poisson Clariidae de Sangalkam (Sénégal)
}

\author{
par G. VASSILIADES, \\ Laboratoire national de Recherches vétérinaires - B.P. 2057, Dakar (Sénégal) \\ Institut d'Elevage et de Médecine vétérinaire des Pays tropicaux, 10, rue Pierre-Curie \\ F 94 -Maisons-Alfort
}

\begin{abstract}
Résumé
Paracamallanus senegalensis n. sp. est parasite de Clarias senegalensis au Sénégal. Ce Nématode se distingue des espèces déjà connues par l'existence d'une capsule buccale et d'un pharynx de dimensions sensiblement égales, de deux tridents chitinoïdes médians de très grande taille, d'un seul spicule, et d'un petit gubernaculum.
\end{abstract}

\section{Summary}

A new species: Paracamallanus senegalensis n. sp. parasite of Clarias senegalensis in Senegal is described. Among already known species we may distinguish this Nematode by the similar size of the buccal capsule and the pharynx, by the large size of the two tridents and by the presence of a unic spicule and a small gubernaculum.

$\mathrm{Au}$ cours d'une étude sur les helminthes parasites des poissons de la rivière de Sangalkam, nous avons pu récolter dans l'intestin d'un Clarias quelques nématodes appartenant au genre Paracamallanus. Nous en donnons ci-après la description. 


\section{Matériel étudié.}

Hôte: Clarias senegalensis Valenciennes, 1840 (famille des Clariidae, sous-ordre des Siluroidei, Téléostéens, Cypriniformes).

Lieu de récolte : rivière de Sangalkam (Rufisque, Région du Cap-Vert, Sénégal) le 7 janvier 1970 .

Localisation des parasites: intestin moyen.

Matériel récolté : 11 femelles et 3 mâles, mis en collection au laboratoire d'helminthologie du Laboratoire national de Recherches vétérinaires de Dakar sous le numéro A 42. Types: 1 mâle et 1 femelle déposés dans les collections du Muséum national d'Histoire naturelle de Paris (Laboratoire de Zoologie-Vers), sous le numéro $1 \mathrm{BA}$.

\section{Description.}

Les nématodes étudiés sont des vers fins, amincis aux deux extrémités et de taille relativement petite, la femelle étant deux fois plus grande que le mâle.

Ils présentent une capsule buccale formée par deux valves latérales et pourvue de deux tridents chitinoïdes, et une cavité buccale ou pharynx située en arrière des valves.

Cuticule fine, légèrement striée.

Les quatre papilles sub-médianes du cycle externe sont grosses et bien visibles, alors que les deux amphides latérales sont difficilement distinctes (Cf. fig. 1, C).

L'œsophage est en deux parties: un œsophage musculaire antérieur, légèrement renflé en massue à sa base avec dans sa lumière trois baguettes chitinoïdes longitudinales, suivi d'un œsophage de structure glandulaire, légèrement plus long. L'anneau nerveux est situé à proximité de la partie antérieure de l'œsophage musculaire; à peu près au même niveau se situent également les deux déirides latérales. Le pore excréteur est nettement plus postérieur, un peu en avant de la jonction des deux portions œsophagiennes (Cf. fig. I, A).

Femelle vivipare, vulve équatoriale. Mâle possédant un seul spicule et un gubernaculum.

DesCRIPTION DE LA RÉGION BUCCO-PHARYNGIENNE (Cf. fig. I, B, C, D).

Capsule buccale à parois épaisses, formée par deux valves latérales articulées ; chaque valve présente:

- une paire de petites pièces chitinoïdes triangulaires $(18 \times 22 \mu)$ situées à la partie antérieure,

- neuf baguettes chitineuses, longitudinales, lisses et de longueurs inégales.

La capsule buccale porte deux tridents chitinoïdes médians, un dorsal et un ventral, comprenant chacun une partie basale large et trois épines très allongées, 

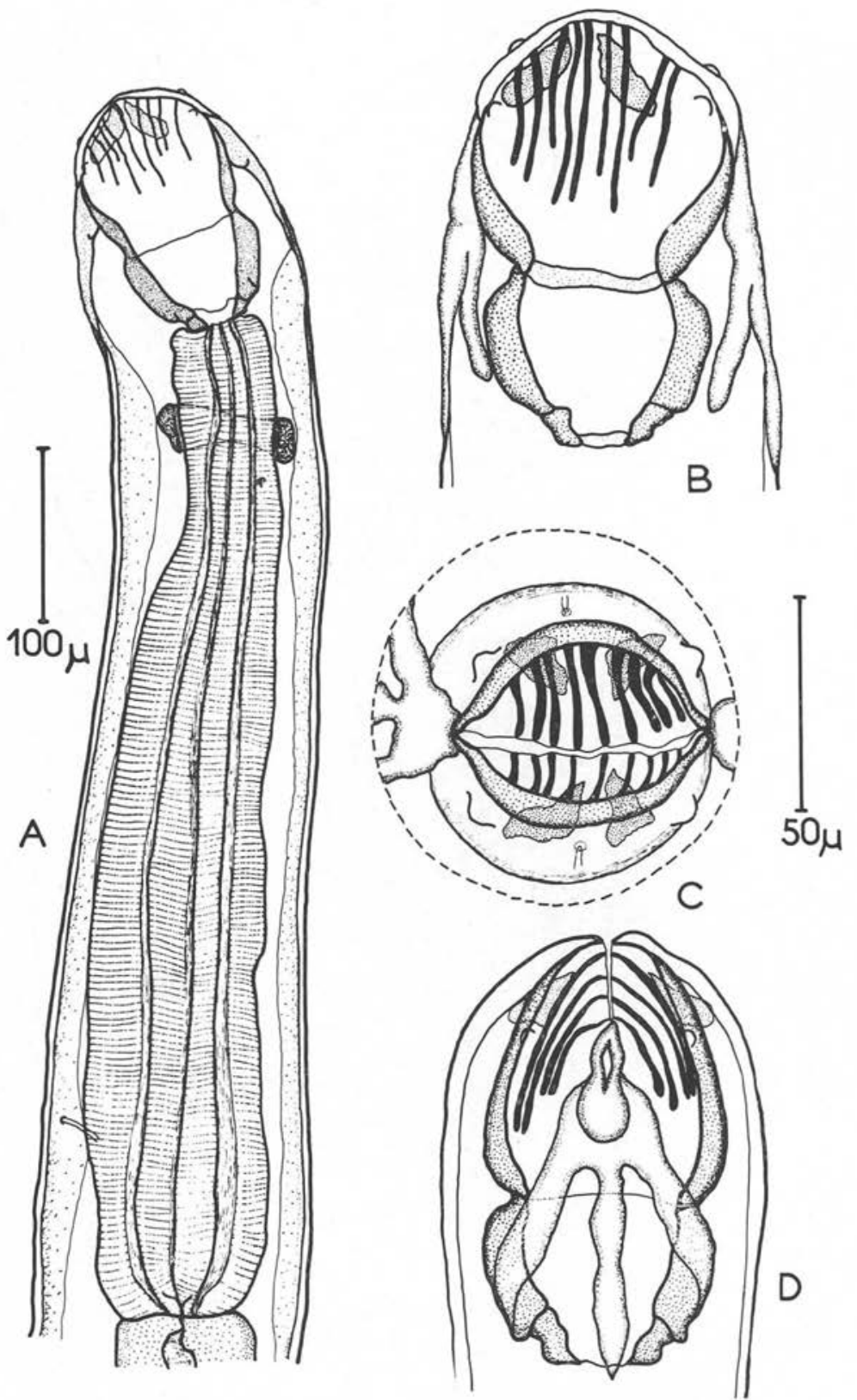

FIG. 1. - Paracamallanus senegalensis n. sp.

A : extrémité antérieure de la femelle, vue latérale (échelle $100 \mu$ ); B : région buccopharyngienne du mâle, vue latérale; $C$ : région bucco-pharyngienne de la femelle, vue apicale ; D : région bucco-pharyngienne du mâle, vue médiane ; $(B, C, D$, échelle $50 \mu)$ 


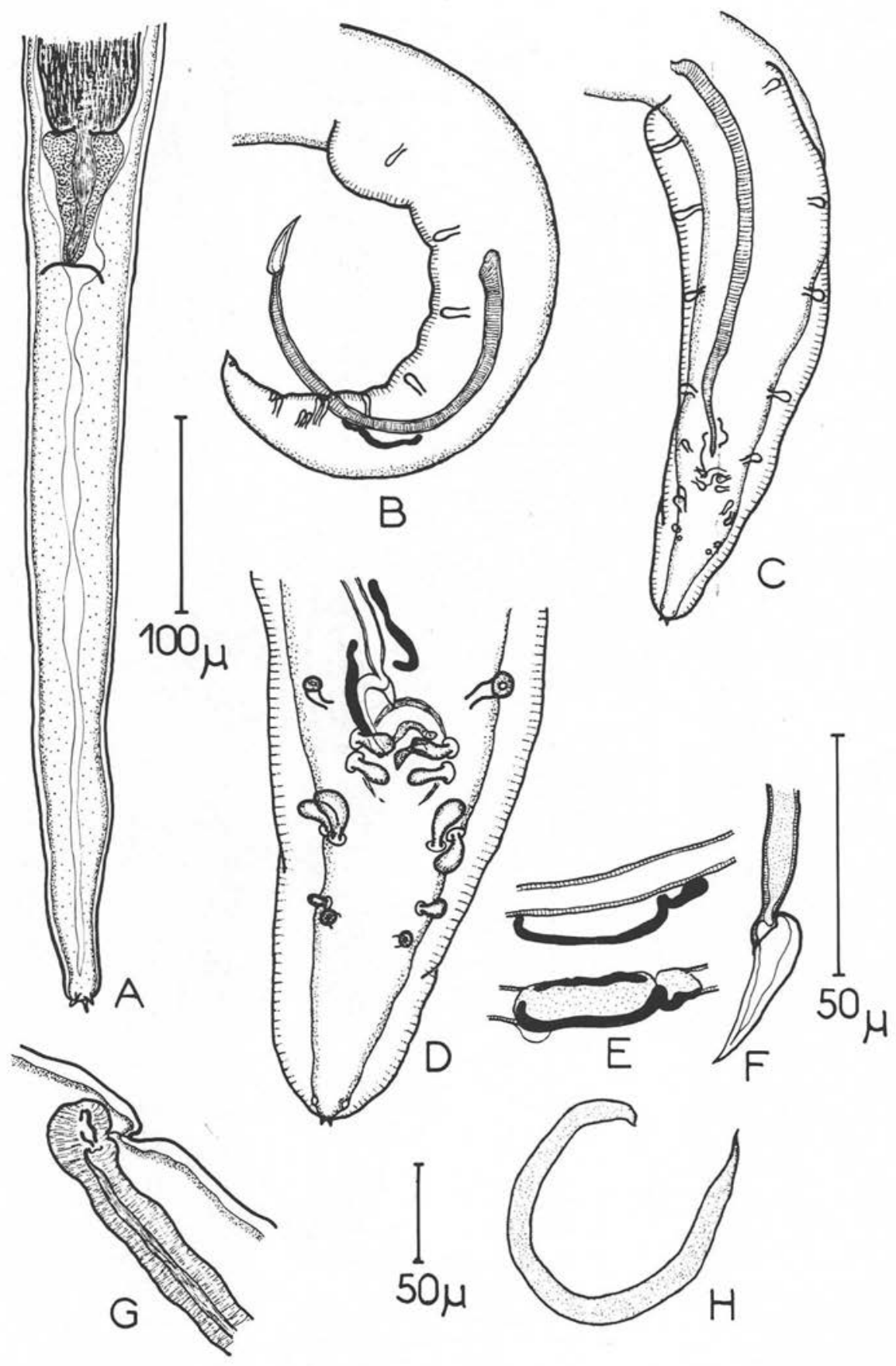


toutes dirigées postérieurement, deux épines latérales et une épine médiane plus longue descendant en arrière jusqu'au niveau de l'œsophage musculaire.

Cavité buccale ou pharynx à parois plus épaisses mais de dimensions légèrement plus réduites.

\section{DESCRIPTION DU MALE.}

Corps long de $5 \mathrm{~mm}$ sur $0,110 \mathrm{~mm}$ de large dans sa partie médiane (toutes les dimensions suivantes se rapportent à cet exemplaire).

Capsule buccale mesurant $60 \mu$ de hauteur comme de largeur ; pharynx : $40 \mu$ sur $50 \mu$ de large ; hauteur totale de la région bucco-pharyngienne : $100 \mu$. Dimensions du trident: partie basale large de $25 \mu$, longueur totale : $75 \mu$ (épine médiane : $45 \mu$.).

Cuticule épaisse de 3 à $4 \mu$, interstries égales à 5 à $6 \mu$.

Anneau nervęux, déirides et pore excréteur respectivement situés à $135 \mu, 165 \mu$. et $525 \mu$ de l'extrémité antérieure.

Esophage musculaire et œsophage glandulaire respectivement longs de $475 \mu$. et $600 \mu$. Longueur totale de l'œsophage égale à $1.075 \mu$.

Queue incurvée ventralement, courte, terminée par deux petites pointes caudales. Anus à $80 \mu$ de l'extrémité postérieure. Ailes caudales bien développées, longues de $300 \mu$ (Cf. fig. II, B, C).

Spicule droit étroit et effilé, long de $270 \mu$, constitué par un manche ou spicule proprement dit long de $240 \mu$ terminé par une sorte de griffe ou de crochet supportant une aile foliacée longue de $30 \mu$ (Cf. fig. II, B, F).

Spicule gauche absent.

Gubernaculum à peine plus large que le spicule qu'il recouvre dorsalement, long de $40 \mu$ et constitué en fait par deux petites pièces, l'une de $30 \mu$, l'autre lui faisant suite, de $10 \mu$. (Cf. fig. II, E).

Douze paires de papilles caudales disposées comme suit :

- cinq paires de papilles préanales à peu près équidistantes, pédonculées,

- deux paires de papilles adanales proches de l'ouverture cloacale, également pédonculées,

- cinq paires de papilles postanales, les quatre premières sont groupées deux à deux, les deux premières paires étant semblables aux papilles préanales, la dernière paire, non pédonculée est difficile à distinguer tout à fait à l'extrémité postérieure.

\section{Ci-contre: FIG. 2. - Paracamallanus senegalensis n. sp.}

A : queue de la femelle, vue ventrale; B: queue du mâle, vue latérale; $\mathrm{C}$ : queue du mâle, vue ventrale; (A, B, C, échelle $100 \mathrm{\omega}$, D : extrémité de la queue du mâle, vuə ventrale; E: gubernaculum, vues dorsale et latérale; F: extrémité distale du spicule; (D, E, F, échelle $50 \mu$ ), $\mathrm{G}$ : vulve de la femelle; $\mathrm{H}$ : larve; $(\mathrm{G}, \mathrm{H}$, échelle $50 \mathrm{\mu})$ 
DESCRIPTION DE LA FEMELLE.

Corps long de $10 \mathrm{~mm}$ sur $0,150 \mathrm{~mm}$ de large vers le milieu du corps (toutes les dimensions données se rapportent à cet exemplaire).

Capsule buccale mesurant $85 \mu$ sur $100 \mu$ de large, pharynx : $55 \mu$ sur $80 \mu$ de large, hauteur totale de la région bucco-pharyngienne : $140 \mu$. Dimensions du trident : partie basale large de $35 \mu$, longueur totale : $110 \mu$ (épine médiane : $60 \mu$ ).

Cuticule épaisse de 4 à $5 \mu$; interstries égales à 6 à $7 \mu$.

Anneau nerveux, déirides et pore excréteur respectivement situés à $200 \mu, 230 \mu$ ct $650 \mu$ de l'extrémité antérieure.

Esophage musculaire et $œ$ sophage glandulaire longs respectivement de $580 \mu$ et $725 \mu$, longueur totale de l'œsophage égale à $1.305 \mu$.

Queue effilée, extrémité arrondie présentant quatre petites pointes caudales. Anus à $400 \mu$ de l'extrémité postérieure (Cf. fig. II, A).

Femelle vivipare, vulve située au milieu du corps, à $5 \mathrm{~mm}$ de l'apex, présentant une lèvre antérieure constituée par un repli cuticulaire proéminent. Vagin à parois musculaires épaisses dirigé postérieurement et long de $500 \mu$. (Cf. fig. II, G). Deux utérus à parois très minces renfermant un grand nombre de larves. Dimensions des larves au niveau de la vulve : $250 \mu$ sur $15 \mu$ de large (Cf. fig. II, H).

\section{Discussion.}

Par les caractères très particuliers de leur extrémité buccale :

- capsule buccale séparée en deux valves et pourvue de deux tridents médians,

- présence d'une cavité buccale en arrière des valves,

les nématodes que nous venons de décrire appartiennent au genre Paracamallanus Yorke et Maplestone, 1926 (Camallanidae, Railliet et Henry, 1917).

Le genre Paracamallanus comprend actuellement, à notre connaissance, trois espèces :

- Paracamallanus cyathopharynx (Baylis, 1923) (=Camallanus cyathopharynx), chez Heterobranchus anguillaris et Clarias parvimanus (Poissons Clarridae), d'Egypte ;

- Paracamallanus sweeti (Moorthy, 1937) (=Camallanus sweeti), chez Ophiocephalus gachua (Poisson Ophiocephalidae), des Indes;

- Paracamallanus ophiocephali Karve, 1941, également chez Ophiocephalus gachua des Indes.

Notre Paracamallanus ne peut être rattaché à aucune de ces trois espèces.

Avec $P$. cyathopharynx, bien que l'auteur ne donne pas une description précise de l'extrémité caudale du mâle, il nous est possible de constater que cette espèce diffère de la nôtre par des caractères concernant la capsule buccale. En effet, outre le fait que les deux paires de petites pièces triangulaires chitinoïdes des valves ne sont pas signalées chez $P$. cyathopharynx dans la description ni dans la figuration originales, chez 
cette dernière espèce les tridents médians sont très petits et débordent à peine sur le pharynx, alors que chez notre espèce ils le recouvrent et même le dépassent.

Avec $P$. sweeti les différences morphologiques sont d'autant plus nettes que cette espèce est considérée comme intermédiaire entre les genres Camallanus et Paracamallanus. Les principales différences avec notre espèce sont les suivantes:

- pharynx relativement peu volumineux,

— baguettes des valves perlées ( 8 à 10 perles par baguette),

- pore excréteur au niveau de l'anneau nerveux,

- présence de deux spicules et absence de gubernaculum,

- présence d'une ventouse caudale chez le mâle.

Pour ce qui concerne $P$. ophiocephali, il ne nous a pas été possible de prendre connaissance de sa description originale. Cependant d'après la figure reproduite dans Yamaguti (1961, II, pl. 5, fig. 35) représentant l'extrémité antérieure du mâle, il nous est possible de constater que chez $P$. ophiocephali le pharynx semble une fois et demie plus grand que la capsule buccale, alors que chez notre espèce la capsule buccale est toujours de dimensions plus grandes ou au moins égales à celles du pharynx. De plus, les baguettes chitinoïdes des valves ne semblent pas lisses comme chez nos exemplaires mais présentent au moins un petit bourrelet chacune. Compte tenu également des différences d'hôtes et de localisation, il ne nous est pas possible de rattacher notre espèce à $P$. ophiocephali.

Pour toutes ces raisons nous pensons que notre espèce est nouvelle et nous proposons de la nommer Paracamallanus senegalensis n. sp.

\section{Bibiographie}

Baylis (H. A.), 1923. - Report on a collection of Parasitic Nematodes mainly from Egypt. Part III. Camallanidae, etc..., Parasitology, 15, (1), 37.

Moorthy (V. N.), 1937. - Camallanus sweeti n. sp., a new species of Camallanidae (Nematoda). J. Parasit., 23 (3), 302-306.

KARVE (J. N.), 1941. - Some parasitic nematodes of fishes. I. J. Univ. Bombay n.s. 10 (3), Biol. Sc. (10), 9-42 (non consulté).

Yamaguti (S.), 1961. - Systema Helminthum. Vol. III. The Nematodes of Vertebrates, Interscience Publishers, Inc., New York (1.261 pp.). 